Fermi National Accelerator Laboratory

FERMIIAB-Conf-93/212-E

CDF

\title{
Top Dilepton Search at CDF
}

The CDF Collaboration

Fermi National Accelerator Laboratory

P.O. Box 500, Batavia, Illinois 60510

August 1993

Submitted to the International Symposium on Lepton and Photon Interactions, Cornell University, Ithaca, New York, August 10-15, 1993 


\section{Disclaimer}

This report was prepared as an account of work sponsored by an agency of the United States Government. Neither the United States Government nor any agency thereof, nor any of their employees, makes any warranty, express or implied, or assumes any legal liability or responsibility for the accuracy, completeness, or usefulness of any information, apparatus, product, or process disclosed, or represents that its use would not infringe privately owned rights. Reference herein to any specific commercial product, process, or service by trade name, trademark, manufacturer, or otherwise, does not necessarily constitute or imply its endorsement, recommendation, or favoring by the United States Government or any agency thereof. The views and opinions of authors expressed herein do not necessarily state or reflect those of the United States Government or any agency thereof. 


\section{Top Dilepton Search at CDF}

by the CDF Collaboration

Abstract

We present preliminary results from a search for the top quark in the dilepton channel using data collected by CDF during the 1992-93 Fermilab Collider Run. The dilepton analysis implies a lower limit on the top quark mass of $113 \mathrm{GeV} / \mathrm{c}^{2}$ at the $95 \%$ confidence level, assuming Standard Model decays.

The Fermilab $p \bar{p}$ collider has recently completed the 1992-93 run, during which $21.4 \mathrm{pb}^{-1}$ of data were collected by the CDF experiment. During the preceding run in 1998-89, CDF collected $4.1 \mathrm{pb}^{-1}$ and set a lower limit of $85 \mathrm{GeV}$ on $M_{\text {top }}$ from the dilepton channel alone. When combined with the results from the lepton + jets $+b$ channel, where the $b$ was tagged through its semileptonic decay into muons, an improved limit of $91 \mathrm{GeV}$ at the $95 \%$ confidence level was obtained. These analyses are described in detail in $[1,2]$. In the following paper we describe the dilepton analysis for the new data from 1992-93.

Top quarks are expected to be produced at the Fermilab collider mainly via the process $p \bar{p} \rightarrow t \bar{t}$, and then to decay into a $W$ boson and a bottom quark, $t \rightarrow W b$. The $W$ decays into a pair of quarks $(u \bar{d}$ or $c \bar{s})$ or into leptons $(e \nu, \mu \nu$, or $\tau \nu)$. The final states of the top quark decay are either three jets or a jet accompanied by a charged lepton and a neutrino. Due to large QCD multijet backgrounds, useful top quark signatures must employ at least one lepton $(e$ or $\mu$ ). The signature used here is two high- $P_{T}$ leptons. Most of the acceptance comes from leptons from $\mathrm{W}$-decay, but electrons and muons from $b$ or $\tau$-decay are also accepted.

CDF is a solenoidal detector with good electron and muon identification capabilities. Electromagnetic and hadronic calorimeters with projective towers cover nearly the full solid angle. Inside the region $|\eta|<1.2$ the central tracking chamber (CTC) measures charged particle momenta with precision $\delta P_{T} / P_{T}^{2} \simeq 0.0011(\mathrm{GeV} / \mathrm{c})^{-1}$. A vertex time projection chamber (VTX) located between the beam pipe and the central tracking chamber provides tracking information out to $|\eta|=3.25$. Electrons are identified in the rapidity regions $|\eta|<1.0$ (central calorimeter) and $1.26<|\eta|<2.2$ (plug calorimeter). Electron candidates have calorimeter clusters with mostly electromagnetic energy and with lateral shower profiles consistent with test beam electrons. They must be associated with a track extrapolating to the calorimeter shower position. For central electrons, the track momentum must be in good agreement with the calorimeter energy. In the plug region, where the CTC resolution and efficiency are degraded, energy-momentum matching is not required, and tracks in the VTX are also used for position matching. Electron pairs from photon conversions and Dalitz decays can be rejected if a second nearby track forming a low mass pair is found in the CTC. Photon conversions can also be rejected if no track is found in the VTX. Muons are identified in the region $|\eta|<1.2$ by requiring that the tower to which the candidate track extrapolates has energy deposition consistent with that of a minimum ionizing particle. The region $|\eta|<1.0$ is instrumented with muon chambers, outside of the calorimeters, for triggering and improved muon identification.

Submitted International Symposium on Lepton and Photon Interactions, Cornel1 University, Ithaca, NY, August 10-15, 1993 
After the lepton identification cuts and a transverse momentum cut, $P_{T}>20$ $\mathrm{GeV} / c$, on both leptons, there are $9 e \mu, 1141 e e$, and $547 \mu \mu$ events in the 1992-93 data. Kinematic and event topology cuts are then applied to reject backgrounds. A back-to-back cut, requiring $\Delta \phi_{\ell \ell}<160$ degrees, where $\Delta \phi_{\ell \ell}$ is the dilepton azimuthal opening angle, and a missing $E_{T}$ cut, $E_{T}>25 \mathrm{GeV}$, reduce Drell-Yan, $b \bar{b}$, and hadron misidentification backgrounds. An additional dilepton invariant mass cut around the $Z^{0}$ peak, $70<M_{\ell \ell}<110 \mathrm{GeV} / c^{2}$, is used for dielectrons and dimuons. In $t \bar{t}$ dilepton events there would be two undetected high transverse energy neutrinos, and the two leptons are not expected to be back-to-back. Therefore, with these cuts we expect good $t \bar{t}$ acceptance.

Of the $1141 e e$ and $547 \mu \mu$ events, $77 e e$ and $54 \mu \mu$ events survive the invariant mass cut (see Figure 1). After imposing the $\Delta \phi_{\ell \ell}$ and $\boldsymbol{E}_{T}$ cuts, one dielectron event remains in the data. Two of the nine $e \mu$ events pass the $\Delta \phi_{\ell \ell}$ and $\boldsymbol{F}_{T}$ cuts (see Figure 2).

The detection efficiencies for the dilepton analysis are summarized in Table 1. The observed cross section is related to the $t \bar{t}$ production cross section as follows : $\sigma_{\text {obs }}=\sigma_{t \bar{t}} B \epsilon_{\text {total }}$, where $\mathrm{B}=\frac{4}{81}$ is the semileptonic branching fraction into ee, $\mu \mu$, or $\mathrm{e} \mu$. The total efficiency is decomposed into several parts and written as $\epsilon_{\text {total }}=$ $\epsilon_{\text {geom. }} P_{T} \epsilon_{I s o l} \epsilon_{I D} \epsilon_{\text {event }} \epsilon_{\text {trigger. }}$. As done in reference [2], we use the Monte Carlo to determine the geometric and kinematic acceptance $\epsilon_{\text {geom }} \cdot P_{\mathrm{T}}$, the efficiency of the isolation cuts $\epsilon_{\mathrm{Isol}}$, and the efficiency of the topology cuts $\left(\mathbf{M}_{\boldsymbol{U}}, \bar{E}_{T}, \Delta \phi\right) \epsilon_{\text {event }}$. The efficiency of the lepton identification cuts $\epsilon_{I D}$ is determined from $Z^{0} \rightarrow e e$ and $Z^{0} \rightarrow \mu \mu$ data. The trigger efficiencies $\epsilon_{\text {trigger }}$ are determined using data collected by independent triggers.

For high mass top events the two $b$ quarks can deposit considerable energy in the calorimeters. A better separation between signal and backgrounds can be obtained by requiring two jets in the region $|\eta|<2.4$ with observed calorimeter transverse energies $E_{T}>10 \mathrm{GeV}$. The efficiency of the two-jet cut $\epsilon_{t w o-j e t}$ is listed as a function of $M_{\text {top }}$ in Table 1, and is included in the total detection efficiency of an optional second set of cuts, $\epsilon_{\text {total }}^{I I}$. Of the three events in the signal region, two of them (the two e $\mu$ events) satisfy the two-jet requirement.

The backgrounds in the dilepton channel are summarized in Table 2. Backgrounds from particle misidentification (fakes) are estimated by measuring the probabilities for tracks or calorimeter clusters from a jet background sample to satisfy muon or electron identification cuts. The fake probabilities are then applied to the number of events in the data with a lepton + track or cluster. Heavy flavor backgrounds, mostly $b \bar{b}$, are studied by using the ISAJET Monte Carlo to get rejection factors for the $P_{T}$ cuts, event topology cuts, and two-jet cuts. The $b \bar{b}$ Monte Carlo yields for lower momentum dileptons $\left(P_{T}^{1}>15 \mathrm{GeV} / c\right.$ and $\left.P_{T}^{2}>5 \mathrm{GeV} / c\right)$ are then normalized to the number of such events found in the data. $Z \rightarrow \tau \tau \rightarrow e \mu$, ee, or $\mu \mu$ are studied using a data sample of $Z \rightarrow$ ee events and substituting the electrons by Monte Carlo $\tau$ 's which are then decayed into $e$ or $\mu$. Similarly, Drell-Yan dielectron and dimuon backgrounds from the continuum, are studied by using $Z$ data to obtain rejection factors for $P_{T}$ cuts, event topology cuts, and two-jet cuts and using the observed continuum mass spectrum for isolated dilepton events. For estimating the dilepton backgrounds from $W W$ and $W Z$ diboson production, we used the ISAJET Monte Carlo. Also shown in Table 2 is the total number of events expected, $N_{e v}=\sigma_{\text {obe }} \int \mathcal{L} d t$, where $\int \mathcal{L} d t$ is the integrated luminosity of the 1992-93 data sample.

Given three candidate events, and without subtracting backgrounds, we derive an 
upper limit on the $t \bar{t}$ production cross section as a function of $M_{\text {top }}$. This upper limit cross section is compared to theoretical lower estimates of $\sigma_{t i}[3]$ to obtain a lower limit on the top quark mass of $108 \mathrm{GeV} / \mathrm{c}^{2}$ at the $95 \% \mathrm{CL}$ based on $21.4 \mathrm{pb}^{-1}$ of data collected in 1992-93. We note that this limit is obtained without using a two-jet cut. Had a two-jet cut been used, with two candidate events instead of three, an inferior limit would have been obtained due to reduced efficiency and additional systematic uncertainties in the jet counting for low mass top. When combining the newer dataset with the $4.1 \mathrm{pb}^{-1}$ collected in $1988-89^{1}$, the lower limit obtained on $M_{\text {top }}$ is $113 \mathrm{GeV} / \mathrm{c}^{2}$ at the $95 \%$ C.L. (see Figure 3).

\footnotetext{
${ }^{1}$ The limit from the combined 1988-89 and 1992-93 datasets is aleo based on three events, without

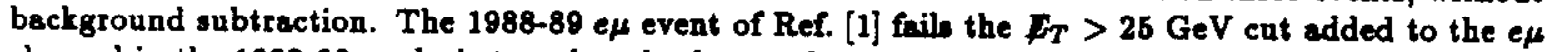
channel in the 1992-93 analyais to reduce backgrounds expected in the larger luminosity data aample.
} 


\begin{tabular}{crrrrrrrr}
\hline$M_{\text {top }}$ & $\epsilon_{\text {geom }} \cdot \mathrm{P}_{\mathrm{T}}$ & $\epsilon_{\text {Isol }}$ & $\epsilon_{\mathrm{ID}}$ & $\epsilon_{\text {event }}$ & $\epsilon_{\text {Trigger }}$ & $\epsilon_{\text {two-jet }}$ & $\epsilon_{\text {total }}^{I}$ & $\epsilon_{\text {total }}^{I I}$ \\
\hline 100 & 0.38 & 0.92 & 0.88 & 0.60 & 0.97 & 0.63 & 0.16 & 0.10 \\
120 & 0.41 & 0.92 & 0.88 & 0.61 & 0.97 & 0.74 & 0.19 & 0.14 \\
140 & 0.53 & 0.91 & 0.88 & 0.61 & 0.97 & 0.81 & 0.24 & 0.20 \\
160 & 0.63 & 0.90 & 0.88 & 0.62 & 0.97 & 0.84 & 0.29 & 0.24 \\
\hline \hline
\end{tabular}

Table 1: Dilepton detection efficiencies. There are two sets of cuts considered. For the first set, $\epsilon_{\text {total }}^{I}=\epsilon_{\text {geom }} \cdot P_{\mathrm{r}} \epsilon_{I s d} \epsilon_{I D} \epsilon_{\text {event }} \epsilon_{\text {trigger. }}$. There is an additional two-jet requirement for the second set of cuts : $\epsilon_{\text {total }}^{I I}=\epsilon_{\text {total }}^{I} \epsilon_{\text {two-jet }}$.

\begin{tabular}{crr}
\hline & Cuts I & Cuts II \\
\hline fakes & $1.85 \pm 0.31$ & $0.40 \pm 0.13$ \\
$b \bar{b}$ & $0.38 \pm 0.20$ & $0.16 \pm 0.09$ \\
$Z \rightarrow \tau \tau$ & $0.64 \pm 0.12$ & $0.24 \pm 0.08$ \\
$W W$ & $0.73 \pm 0.22$ & $0.16 \pm 0.05$ \\
$W Z$ & $0.07 \pm 0.02$ & $0.02 \pm 0.01$ \\
Dreil-Yan & $0.80 \pm 0.23$ & $0.29 \pm 0.14$ \\
\hline Total Bg. & $4.5 \pm 0.5$ & $1.3 \pm 0.2$ \\
\hline CDF Data & 3 & 2 \\
\hline$M_{\text {top }}=100$ & 14 & 9.1 \\
$M_{\text {top }}=120$ & 6.4 & 5.0 \\
$M_{\text {top }}=140$ & 3.7 & 3.1 \\
$M_{\text {top }}=160$ & 2.2 & 1.8 \\
\hline \hline
\end{tabular}

Table 2: Numbers of background events, data yields, and expected signal for the 199293 run. The first column (Cuts I) lists the numbers of events after the $\mathrm{M}_{\boldsymbol{U}}, \boldsymbol{E}_{T}$ and $\Delta \phi$ event topology cuts. For the second column ( Cuts II), there is an additional two-jet requirement. 

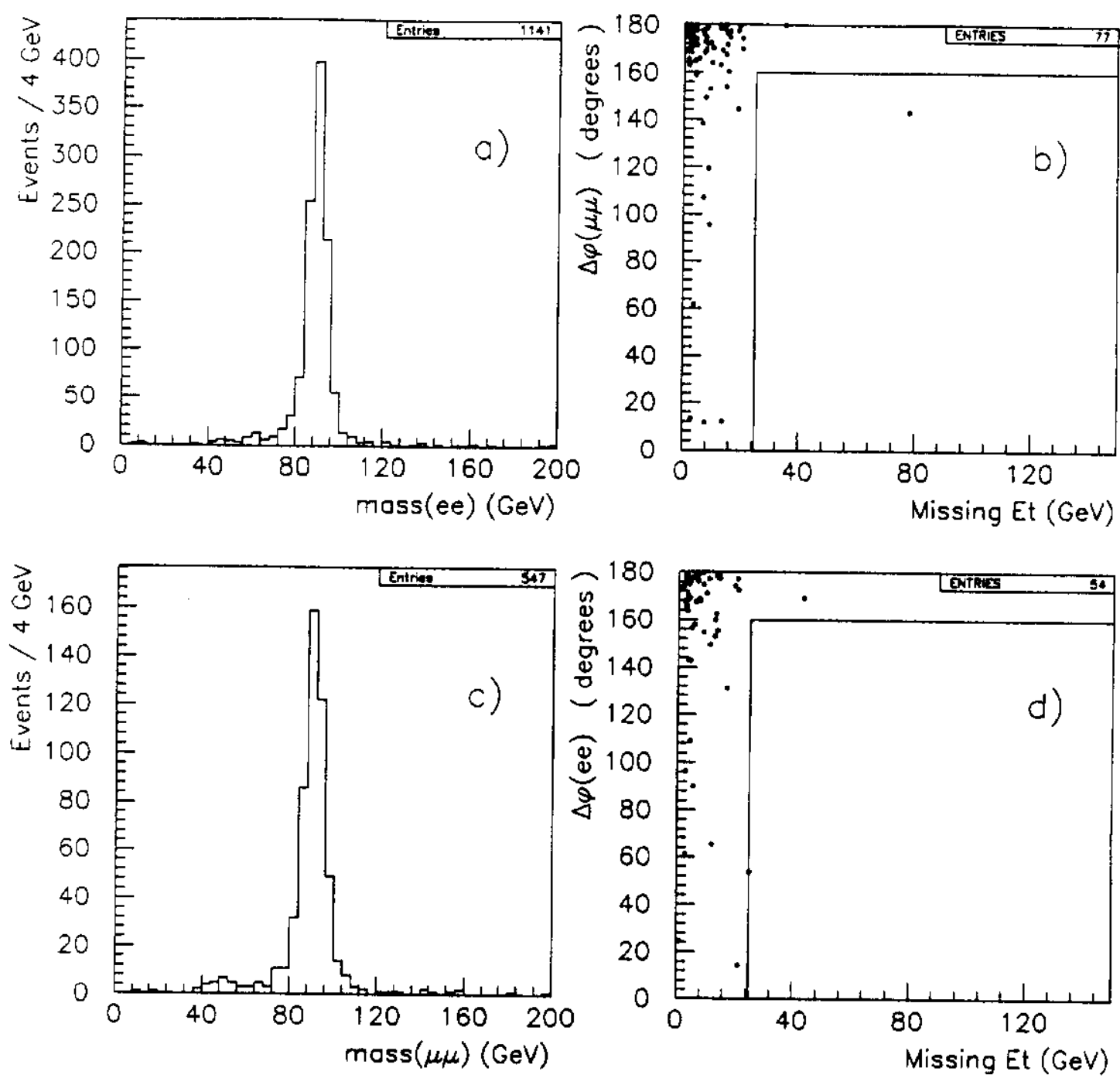

Figure 1: Distributions for CDF ee and $\mu \mu$ data for the 1992-93 run. There is one $e e$ event in the rignal region. a) Dielectron Invariant Mass b) $\Delta \phi$ vs missing $E_{\mathrm{T}}$ for dielectrons surviving the $Z^{0}$ mass window cut c) Dimuon Invariant Mass d) $\Delta \phi$ vs missing $\mathrm{E}_{\mathrm{T}}$ for dimuons surviving the $\mathrm{Z}^{0}$ mass window cut 

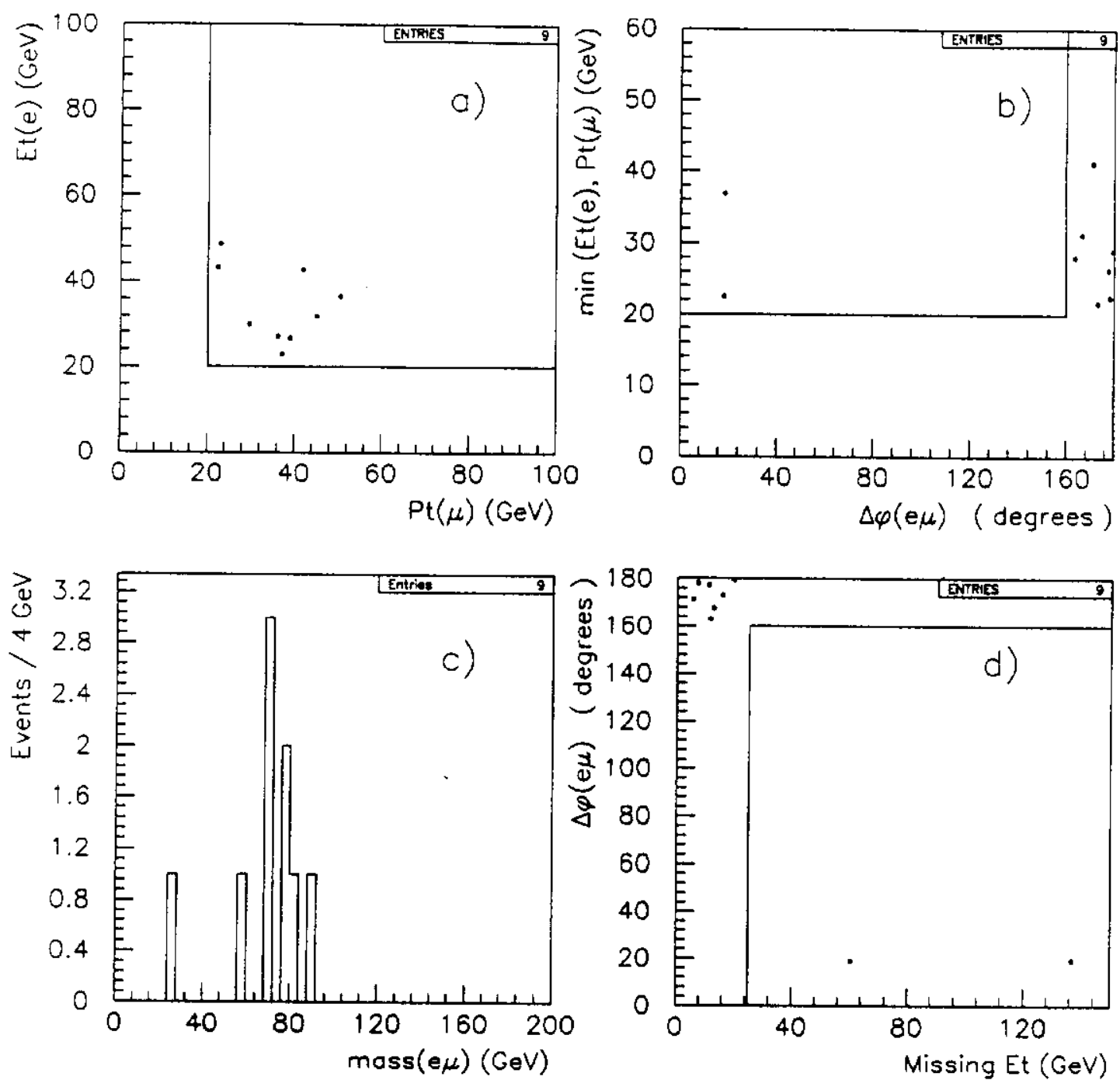

Figure 2: Distributions for CDF e $\mu$ data for the 1992-93 run. There are two e $\mu$ events in the signal region. a) $\mathrm{E}_{\mathrm{T}}(\mathrm{c})$ vs $\mathrm{P}_{\mathrm{T}}(\mu)$ b) $\operatorname{Min}\left(\mathrm{E}_{\mathrm{T}}, \mathrm{P}_{\mathrm{T}}\right)$ vs $\Delta \phi \mathrm{c}$ ) Invariant mass of $e \mu$ pairs d) $\Delta \phi$ vs missing $\mathrm{E}_{\mathrm{T}}$ 


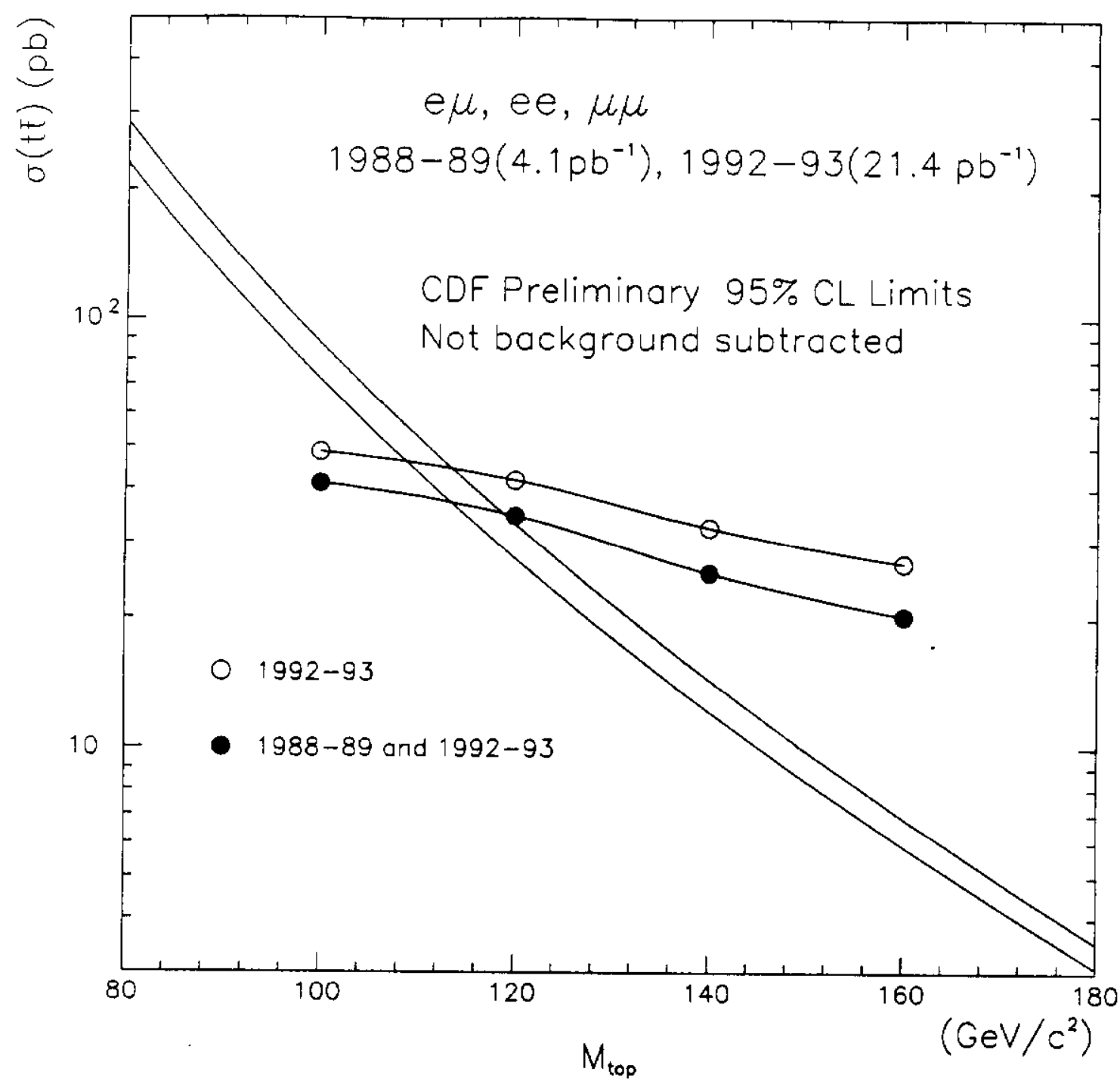

Figure 3: Upper limits at the 95\%-C.L. on $\sigma_{t \bar{t}}$ compared with the lower bound and the central value of the theoretical prediction from Ref. [3]. The two sets of experimental limits are: (1) from the 1992-93 dilepton data alone; (2) from the combination of the 1988-89 and 1992-93 dilepton data. 


\section{References}

[1] F. Abe et al., A Lower Limit on the Top Quark Mass from Events with Two Leptons in $p \bar{p}$ Collisions at $\sqrt{s}=1.8 \mathrm{TeV}$, by The CDF Collaboration, Physical Review Letters 68, 447-451 (1992)

[2] F. Abe et al., Limit on the Top-Quark Mass from Proton-Antiproton Collisions at $\sqrt{s}=1.8 \mathrm{TeV}$, by The CDF Collaboration, Physical Review D45, 3921-3948 (1992)

[3] P. Nason, S. Dawson and R.K. Ellis, Nucl. Phys. B303, 607 (1988). G. Altarelli, M. Diemoz, G. Martinelli and P. Nason, Nucl. Phys. B308, 724 (1988). R.K. Ellis, Fermilab-Pub-91/30-T. 